\title{
Validade Incremental da Escala de Abordagens de Aprendizagem (EABAP)
}

\author{
Incremental Validity of the Learning Approaches Scale
}

\author{
Cristiano Mauro Assis Gomes* \& Hudson Fernandes Golino \\ Universidade Federal de Minas Gerais, Belo Horizonte, Brasil
}

\begin{abstract}
Resumo
Este trabalho investiga a validade incremental da abordagem à aprendizagem ( $\mathrm{AbA}$ ) sobre o desempenho escolar, além da inteligência. São analisados dados de 684 estudantes da sexta série ao terceiro ano do ensino médio de uma escola particular de Belo Horizonte, Minas Gerais, Brasil. A inteligência é mensurada por itens marcadores da inteligência fluida da Bateria de Fatores Cognitivos de Alta-Ordem. AbA é medida através da Escala de Abordagens de Aprendizagem. O desempenho acadêmico é medido através das notas escolares em Matemática, Português, Geografia e História. Três hipóteses sobre a relação entre inteligência, abordagem à aprendizagem e proficiência acadêmica são testadas através da modelagem por equação estrutural. $\mathrm{O}$ modelo da relação direta foi o mais adequado aos dados e apresentou bom grau de ajuste. Inteligência e $\mathrm{AbA}$ apresentam efeito direto sobre o desempenho escolar. AbA possui validade incremental independente da inteligência sobre as diferenças individuais do rendimento acadêmico.

Palavras-chave: Abordagem superficial, abordagem profunda, inteligência, validade incremental, proficiência acadêmica.
\end{abstract}

\begin{abstract}
This paper investigates the incremental validity of learning approach in academic achievement. Participants were 684 junior and high school students from a private school in Belo Horizonte, Minas Gerais, Brazil. Intelligence is measured by fluid intelligence items of the Higher-Order Cognitive Factors Kit. Learning approach is measured by the Learning Approaches Scale. Academic achievement is measured by annual grades in Mathematics, Portuguese (native language), Geography, and History. Three hypotheses about the relation among intelligence, learning approach and academic achievement are tested through structural equation modeling. The direct relation model was the most adequate and showed good fit. Intelligence and learning approach show direct effect in academic achievement. Learning approach has incremental validity of individual differences in academic achievement, independently of intelligence.

Keywords: Deep approach, surface approach, intelligence, incremental validity, academic achievement.
\end{abstract}

\begin{abstract}
Abordagem à Aprendizagem
A abordagem à aprendizagem $(\mathrm{AbA})$ nasceu como construto psicológico a partir da observação de Marton e Saljö (1976a, 1976b) de que os alunos apresentavam uma abordagem superficial ou uma abordagem profunda quando eram solicitados a ler e compreender textos. A abordagem profunda (AP) indica que a interação do aluno com os objetos de conhecimento é intensa, profunda e de boa qualidade. Já a abordagem superficial (AS) indica que a interação sujeito-objeto é pobre, superficial e de baixa qualidade (Biggs, 1987a; Entwistle \& Ramsden, 1983; Riding \& Rayner, 1998; Struyven, Dochy, Janssens, \&
\end{abstract}

\footnotetext{
*Endereço para correspondência: Departamento de Psicologia, Universidade Federal de Minas Gerais, Av. Antônio Carlos, 6627, Sala 4010, Pampulha, Belo Horizonte, MG, Brasil 31270-901. E-mail: cristianogomes@ufmg.br
}

Gielen, 2006; Vermunt \& Vermetten, 2004; Wild \& Quinn, 1998). O termo abordagem é usado nesta tradição ao invés de estratégia à aprendizagem, na medida em que a abordagem é entendida como uma mistura indissociada de estratégias de aprendizagem e motivações para aprender (Entwistle, McCune, \& Walker, 2001; Entwistle, Meyer, \& Tait, 1991; Entwistle \& Ramsden, 1983; Struyven et al., 2006; Vermunt \& Vermetten, 2004).

Várias baterias foram elaboradas para mensurar a abordagem à aprendizagem (AbA). Dentre elas, encontram-se: o Learning Process Questionnaire (Biggs, 1985, 1987a, 1987b), o Study Process Questionnaire (Biggs, 1978, 1987b), e suas versões reformuladas, o R-SPQ-2F (The Revised Two - Factor Study Process Questionnaire) e o RLPQ-2F (The Revised Two - Factor Learning Process Questionnaire), de Biggs e colaboradores (Biggs, Kember, \& Leung, 2001), o Approaches to Study Inventory, de Entwistle e Ramsden (1983), a Escala de Avaliação de Processos de Estudo (C. F. Gomes, 2005), e a versão em por- 
tuguês do Study Process Questionnaire, denominada Questionário de Processos de Aprendizagem, desenvolvida por Rosário e colaboradores (Rosário, Almeida, Núñez, \& González-Pienda, 2004), entre outros.

Evidências mostram que a AP influencia positivamente o desempenho acadêmico, contribui para uma aprendizagem efetiva e possibilita a superação de dificuldades pessoais e ambientais (Da Silva \& Sá, 1993; Garner, Hare, P. Alexander, Haynes, \& Winograd, 1984; Pressley \& Levin, 1983; Weinstein \& Mayer, 1985). É notório, portanto, a relevância social do estudo da $\mathrm{AbA}$, cujas pesquisas têm contribuído para uma maior e melhor compreensão acerca do desempenho discente, auxiliando na identificação e prevenção de problemas de aprendizagem e intervenções psicoeducacionais (Almeida, 1992, 1996; Biggs, 1985, 1987a; Boruchovitch, 1993, 1999; Entwistle, 1988; Hattie, Biggs, \& Purdue, 1996). Pelas possibilidades apontadas, a literatura da AbA tem fornecido subsídios teóricos e empíricos sobre como o ensino pode melhorar a aprendizagem discente (Biggs \& Tang, 2007; Case \& Marshall, 2009; Fry, Ketteridge, \& Marshall, 2009; Light, Cox, \& Calkins, 2009; Oliver, 2007; Robinson \& Udall, 2006; Wadhwa, 2008; D. A. Watkins, 2007; C. Yu, Williams, Lin, \& W.-C. Yu, 2010).

Discorrendo sobre a literatura nacional, ela apresenta poucos trabalhos sobre o tema (busca realizada em 11 de Abril de 2010, por meios das palavras-chave "abordagens de aprendizagem", "abordagem de aprendizagem", "abordagem à aprendizagem" " "abordagens à aprendizagem"). No banco de dados Scielo não há produções com esses termos. No banco Pepsic, há um artigo de autores estrangeiros. No Google Books nenhum livro foi encontrado com os termos "abordagem à aprendizagem" e "abordagem de aprendizagem". No banco dos currículos Lattes, através da busca por assunto no dia 11 de Abril de 2010, com filtro de busca em doutores, o termo "abordagem à aprendizagem" apresentava quatro pesquisadores e o termo "abordagem de aprendizagem" mostrava 11 pesquisadores. Entrando nos currículos dos pesquisadores, verificou-se que uma parte considerável não trabalhava com o tema ou não publicou artigos científicos sobre o mesmo. Alguns estudos encontrados são Fuentes, Lima e Guerra (2009), que relacionaram as abordagens à aprendizagem ao ensino de matemática no ensino superior, e Souza e Boruchovitch (2009), que incorporaram a análise da AP e AS na discussão da formação de professores.

\section{Validade Incremental}

A inteligência talvez seja o construto psicológico com maior número de evidências sobre sua estrutura e poder preditivo. Dados mostram que ela é um forte preditor acadêmico (Deary, Strand, Smith, \& Fernandes, 2007; Gustafsson \& Undheim, 1996; Naglieri \& Bornstein, 2003; B. Spinath, F. M. Spinath, Harlaar, \& Plomin, 2006), de forma que diferentes modelos teóricos propõem distintas relações entre inteligência e desempenho escolar (Deary \& Johnson, 2010). Ceci (1991), por exemplo, argumenta que ambos são muito próximos porque justamente os conteúdos de suas avaliações são bastante similares. Brody (1997), por sua vez, sustenta que a inteligência tem papel causal sobre as diferenças de desempenho acadêmico, mas observa que uma melhor performance escolar impulsiona a inteligência a novos patamares. Outros modelos, por sua vez, postulam que a inteligência é a base causal das diferenças individuais no desempenho acadêmico (Colom \& Flores-Mendoza, 2007; Gustafsson \& Undheim, 1996; Neisser et al., 1996; M. W. Watkins, Lei, \& Canivez, 2007).

Levando-se em consideração a importância da inteligência e sua força preditiva, a psicologia deve atentar seriamente à recomendação de Gagné e St. Père (2001) de que os pesquisadores devem "examinar o papel causal antecedente da inteligência ao considerarem construtos psicoeducacionais populares, como autoconceito acadêmico, autoeficácia, confiança, auto-regulação, e muitos outros" (Gagné \& St. Père, 2001, p. 96).

Alguns pesquisadores têm adotado como agenda de pesquisas o estudo da validade incremental de variáveis psicológicas, além da inteligência, como preditoras do desempenho acadêmico. Validade incremental, segundo Pasquali (2007) é "a questão de se uma medida particular aporta poder explicativo sobre e além de outra medida para predizer um critério relevante" (p. 100). No campo da motivação há um conjunto relevante de estudos nesta agenda, a partir dos anos 2000 (Beier, Campbell, \& Crook, 2010; Freudenthaler, B. Spinath, \& Neubauer, 2008; Schwinger, Steinmayr, \& B. Spinath, 2009; B. Spinath et al., 2006; F. M. Spinath, B. Spinath, \& Plomin, 2008; Steinmayr \& B. Spinath, 2009). Concomitantemente, os estudos sobre metacognição apresentam uma tendência semelhante (J. M. Alexander, Johnson, Albano, Freygang, \& Scott, 2006; J. M. Alexander, Johnson, Leibham, \& DeBauge, 2004; Lajoie, 2008; Schunk, 2008; Stel \& Veenman, 2008; Veenman \& Beishuizen, 2004; Veenman, Kerseboom, \& Imthorn, 2000; Veenman \& Spaans, 2005).

Pode-se constatar uma situação oposta à encontrada em campos como a motivação e a metacognição, ao se investigar a presença de estudos de validade incremental no campo de estudos em AbA, com um número bastante limitado de estudos dessa natureza (uma busca em 18 de Maio de 2011, com os termos "approaches to learning" e "intelligence", e os termos "learning approach" e "intelligence", encontrou apenas três estudos sobre a validade incremental da $\mathrm{AbA}$ ) no que diz respeito à predição do desempenho escolar, além da inteligência. Os bancos de dados verificados foram: PsycINFO, Academic Search Premier (EBSCO), Cambridge Journals Online, Education Full Text (Wilson), JSTOR Arts \& Sciences I, Collection, Oxford Journals (Oxford University Press), Project Muse, PsycArticles (APA), SAGE Journal Online, SocINDEX with Full Text (EBSCO), SpringerLink (MetaPress), Wiley InterScience.

Diseth (2002) estudou a relação entre habilidades cognitivas e abordagem de aprendizagem em 89 estudan- 
tes do curso de Graduação em Psicologia de uma Universidade da Noruega. Uma medida de inteligência geral foi obtida por meio da análise fatorial de três testes de inteligência: Teste de Vocabulário do WAIS III (Wechsler, 1997), Teste de Analogia Verbal de Mønnesland (1985) e o Teste Espacial de Sandefjord/Rybakoff (Paasche, 1957). A abordagem de aprendizagem foi aferida por meio do Inventário de Abordagens e Habilidades de Estudo (Diseth, 2001; Entwistle, 1997), enquanto o rendimento acadêmico foi aferido por meio de uma prova aberta semestral final. Os resultados apontaram correlações significativas entre abordagem superficial e o Teste de Vocabulário do WAIS ($0,24, p<0,05)$, abordagem superficial e desempenho acadêmico $(-0,20, p<0,05)$, assim como entre este e o Teste de Vocabulário do WAIS $(0,23, p<0,05)$. Os autores tentaram verificar se a abordagem superficial possuía algum poder incremental, além do resultado do Teste de Vocabulário do WAIS, em relação ao desempenho escolar. No entanto, essa análise evidenciou que a abordagem superficial não contribui, além do resultado no Teste de Vocabulário, para a explicação da variância da prova semestral.

Chamorro-Premuzic e Furnham (2008) também estudaram a relação entre abordagem de aprendizagem, inteligência e rendimento acadêmico, além de verificar o papel da personalidade nessa relação. Cento e cinquenta e oito (158) universitários responderam o Questionário de Processos de Estudo (Biggs, 1987b), o Inventário de Personalidade NEO-PI-R (Costa \& McCrae, 1992), o Teste de QI de Wonderlic (1992) e o Teste de Raciocínio de Baddeley (1968). Um ano após responderem aos testes, os estudantes completaram seis exames curriculares da universidade. A média desses seis exames foi utilizada como indicador de desempenho acadêmico. Os resultados apontaram correlações significativas $(p<0,05)$ entre o desempenho acadêmico e as seguintes variáveis: abordagem profunda $(0,33)$, abordagem estratégica $(0,18)$, conscienciosidade $(0,37)$, abertura para experiência $(0,21)$, inteligência geral $(0,24)$ e Gf $(0,27)$. Os resultados da análise de regressão stepwise mostraram que Gf explica cerca de $6 \%$ da variância do desempenho acadêmico, conscienciosidade possui uma explicação adicional de $27 \%$, abertura para experiência possui um valor incremental de $4 \%$, e abordagem profunda adiciona uma explicação de $3 \%$ da variância. Esses dados sugerem que a abordagem profunda possui validade incremental além da inteligência e personalidade, mesmo que o aumento da capacidade de explicação da variância não seja grande.

O último estudo publicado, encontrado na pesquisa aos bancos de dados, descrita anteriormente, foi realizado por Furnham, Monsen, e Ahmetoglu (2009). Duzentos e doze (212) estudantes do ensino básico, com média de idade em 15,8 anos $(D P=0,98)$ completaram o Inventário NEO dos Cinco Fatores (NEO-FFI) de Costa e McCrae (1992), a Escala de Engajamento Intelectual (Goff \& Ackerman, 1992), o Questionário de Processos de Estudo (Biggs, 1987b), o Teste de QI de Wonderlic (1992), e o General Knowledge Test, de Irving, Cammock e Lynn (2001). O resultado no General Certificate in Secondary Education (GCSE), um teste educacional padronizado utilizado na América do Norte e em alguns países da Europa, foi empregado como medida do desempenho acadêmico em Inglês, Matemática, Ciências e Literatura. Os resultados obtidos indicaram que apenas a abordagem estratégica apresentou validade incremental, entre as abordagens de aprendizagem. Ela contribuiu com um incremento de 1\% na explicação da variância tanto do escore em literatura, quanto do escore somado em matemática e ciências, e do escore somado em matemática e inglês. Respectivamente, esses escores tiveram sua variância explicada em $55 \%$, $57 \%, 34 \%$ e $28 \%$.

Dois dos três trabalhos empíricos localizados na pesquisa aos bancos de dados, realizada neste estudo, apresentam evidências iniciais de que as abordagens de aprendizagem possuem validade incremental em relação ao desempenho escolar, além da inteligência e outras variáveis, mesmo que sua carga explicativa seja muito baixa, i.e. entre 1 e 3\%. Essas evidências iniciais são complementares àquelas apresentadas na literatura internacional que apontam a capacidade preditiva da $\mathrm{AbA}$ frente à proficiência acadêmica (Diseth \& Martinsen, 2003; Fenollar, Román, \& Cuestas, 2007; Furnham et al., 2009; Liem, Lau, \& Nie, 2008; Senko \& Miles, 2008). O presente trabalho tem, portanto, como objetivo verificar se a AbA possui validade incremental sobre a explicação do desempenho acadêmico, além da inteligência. Pretende-se investigar, também, qual é o caminho de interação entre $\mathrm{AbA}$ e inteligência, ou seja, se ambas estão em um mesmo nível hierárquico, ou se uma está em posição hierarquicamente superior à outra. Vale salientar que, ao contrário dos estudos relatados anteriormente, não foi inserida nenhuma outra variável psicológica neste estudo, a não ser $\mathrm{AbA} \mathrm{e}$ inteligência. Justifica-se essa escolha uma vez que, sendo a inteligência reconhecida como um dos melhores e mais robustos preditores do rendimento acadêmico (Colom \& Flores-Mendoza, 2007; Deary et al., 2007; Gustafsson \& Undheim, 1996; Naglieri \& Bornstein, 2003; Neisser et al., 1996; B. Spinath et al., 2006; M. W. Watkins et al., 2007), encontrar uma carga explicativa adicional por parte da $\mathrm{AbA}$ se constituiria como uma evidência importante de sua validade incremental.

\section{Hipóteses}

Algumas questões atuais sobre o poder preditivo das variáveis psicológicas vêm sendo traçadas pela literatura internacional. Devido à robustez das evidências sobre inteligência, uma agenda de pesquisas tem se caracterizado por investigar se as variáveis psicológicas, além da inteligência, têm algum papel incremental na predição de aspectos como o desempenho escolar. Essa agenda também investiga como a inteligência se relaciona às outras variáveis psicológicas, em termos de independência ou correlação. O presente estudo pertence a essa agenda de pesquisas e busca verificar as duas questões apontadas, em amostras brasileiras. No entanto, este trabalho procu- 
ra dar um passo adiante e verificar se a relação entre a inteligência e a abordagem à aprendizagem é hierárquica, indicando que uma delas explica indiretamente o desempenho escolar, pois é mediada pela outra variável.
As hipóteses deste estudo são formuladas tendo como referência as questões de investigação expostas. As hipóteses são descritas, a seguir, e sua representação gráfica é exposta na Figura 1.

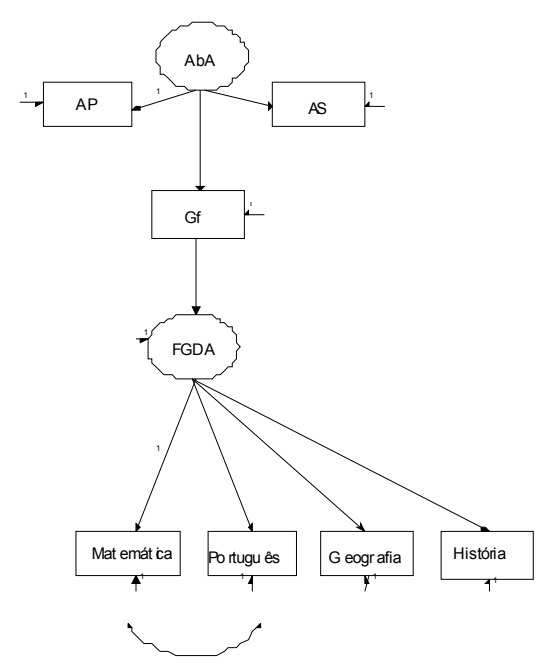

Hipótese 1

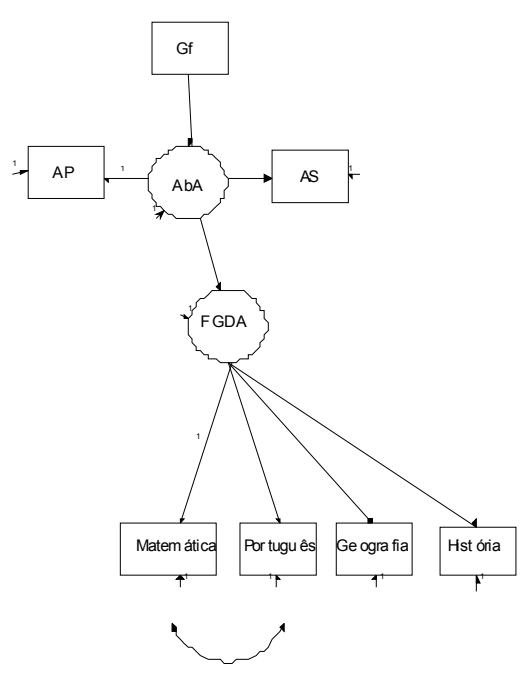

Hipótese 2

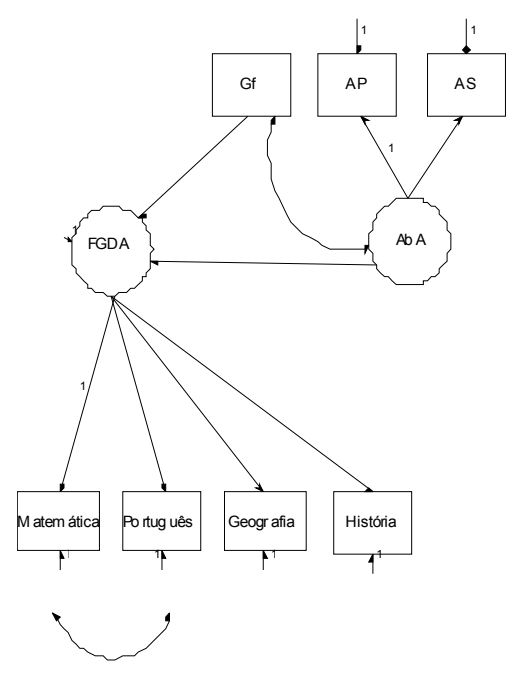

Hipótese 3

Figura 1. Três hipóteses do estudo.

Nota . Legenda: $\mathrm{AbA}=$ Abordagem à aprendizagem; $\mathrm{AP}=$ Abordagem profunda; $\mathrm{AS}=$ Abordagem superficial; $\mathrm{Gf}=\mathrm{Inteligência}$ fluida; FGDA = Fator geral de desempenho acadêmico.

Hipótese 1. Parte das diferenças individuais na inteligência é causada pela abordagem à aprendizagem. A AbA não tem efeito causal direto sobre o fator geral de desempenho acadêmico. Ele é mediado pela inteligência. Parte da variância do fator geral de desempenho acadêmico é causada diretamente pela inteligência.

Hipótese 2. Segue o caminho oposto à hipótese 1. Parte das diferenças individuais presentes na abordagem à aprendizagem é causada pela inteligência. A inteligência não tem efeito causal direto sobre o fator geral de desempenho acadêmico. Ele é mediado pela abordagem à aprendizagem. Parte da variância do fator geral de desempenho acadêmico é causado diretamente pela AbA.

Hipótese 3. Diferentemente das hipóteses anteriores, não há a suposição de uma relação hierárquica causal entre inteligência e abordagem à aprendizagem. Elas são identificadas em uma relação horizontal. Ambas se correlacionam e apresentam um efeito explicativo direto sobre o fator geral de desempenho acadêmico.

\section{Método}

\section{Participantes e Procedimentos}

A amostra é composta por 684 estudantes da sexta série do ensino fundamental ao terceiro ano do ensino médio de uma escola particular de Belo Horizonte, Minas Gerais, Brasil. Os dados foram coletados em 2008. Foram tomados todos os cuidados éticos em relação aos participan- tes e a pesquisa contou com a aprovação de Comitê de Ética. O termo de consentimento livre e esclarecido foi assinado pelos alunos e por seus respectivos responsáveis legais, de acordo com as diretrizes estabelecidas pelo Comitê de Ética da Universidade Federal de Minas Gerais (UFMG). Os instrumentos foram aplicados coletivamente em 19 classes por psicólogos ou estudantes de psicologia devi-damente treinados. A amostra é relativamente equilibrada nas séries $\left(\chi^{2}=0,00 ; g l=6\right.$; sexta série, $N=91$; sétima série, $N=107$; oitava série, $N=98$; nona série, $N=116$; primeiro ano, $N=90$; segundo ano, $N=93$; terceiro ano, $N=89)$, sexo $(N=328$ masculino e $N=356$ feminino $)$ e idade (11 anos, $N=83 ; 12$ anos, $N=106 ; 13$ anos, $N=92 ; 14$ anos, $N=108 ; 15 \operatorname{anos}, N=98 ; 16 \operatorname{anos}, N=80 ; 17 \operatorname{anos}, N=73)$, com exceção do grupo 10 anos $(N=35)$ e 18 anos de idade $(N=9)$. Como os alunos são todos oriundos de uma mesma escola, distribuídos ao longo de várias séries escolares, foi obtida uma amostra relativamente homogênea em termos socioculturais.

\section{Instrumentos}

Conjunto de Testes de Inteligência Fluida (CTIF). Foram utilizados 27 itens do CTIF, todos marcadores de inteligência fluida da Bateria de Fatores Cognitivos de Alta-Ordem (C. M. A. Gomes \& Borges, 2009). Desses itens, oito são do Teste de Indução, 11 são do Teste de Raciocínio Lógico e oito são do Teste de Raciocínio Geral. Evidências mostram que o CTIF apresenta uma confia- 
bilidade adequada (alfa maior ou igual a 0,70 ) e validade convergente e divergente para amostras do ensino fundamental II e ensino médio (C. M. A. Gomes \& Borges, 2009). Os itens foram escolhidos de acordo com suas propriedades psicométricas, ou seja, aqueles que apresentassem maior confiabilidade e carga fatorial no seu fator de referência foram utilizados no presente estudo. Além da confiabilidade e da carga fatorial, os itens foram escolhidos de forma a gerar um conjunto equilibrado, em níveis de dificuldade baixo, médio e alto (C. M. A. Gomes \& Borges, 2009).

Teste de Indução (I). Foram selecionados oito itens com um tempo limite de oito minutos. Cada item é formado por cinco grupos de quatro letras. Cada grupo possui quatro letras que mostram o mesmo padrão. $\mathrm{O}$ respondente deve identificar o grupo que não apresenta o padrão e marcá-lo com um X.

Teste de Raciocínio Lógico (RL). Foram selecionados 11 itens com um tempo restrito de 11 minutos. Cada item é formado por uma conclusão proveniente de duas premissas abstratas. $\mathrm{O}$ respondente deve indicar se a conclusão é logicamente correta.

Teste de Raciocínio Geral (RG). Foram selecionados oito itens com um tempo limite de 12 minutos. Cada item é formado por uma situação-problema matemática e cinco opções de múltipla escolha.

Uma medida de Gf foi obtida através de todos os itens selecionados. A consistência interna dos itens é adequada, com um alfa de 0,75 . A análise fatorial confirmatória mostra que o modelo unidimensional é adequado para explicar a variância dos itens $\left(\chi^{2}=233,56 ; g l=134 ; \mathrm{CFI}=0,90\right.$; RMSEA $=0,03)$. Excetuando os itens RL1 $(0,34), \operatorname{RL} 4(0,27)$, RL5 (0,39), RL9 (-0,05), RL10 (0,23), RL $11(0,39), \operatorname{RG} 1$ $(0,29), \operatorname{RG} 7(0,35)$, todos os outros tiveram carga fatorial maior do que 0,40 no traço latente $\mathrm{Gf}$ (inteligência fluida). As análises foram elaboradas através do software Mplus 5.2. O escore fatorial gerado foi utilizado para a análise de dados das variáveis da questão de estudo.

Escala de Abordagens de Aprendizagem (EABAP). A EABAP é uma escala de auto-relato formada por 17 itens. Cada item possui um enunciado que remete a um comportamento do estudante frente à aprendizagem. $\mathrm{O}$ aluno julga o quanto do comportamento descrito está presente em sua vida, utilizando uma escala do tipo-Likert que varia de (1) nem um pouco até (5) totalmente.

Os itens da EABAP, em geral, articulam uma relação intrínseca entre motivação e estratégia. Os enunciados, majoritariamente, designam tanto estratégias quanto motivações para aprender. Ela pode ser aplicada individual ou coletivamente e não possui limite de tempo, apesar de não demandar mais de 15 minutos para sua realização. Há evidências favoráveis a respeito da validade da estrutura fatorial, validade convergente, validade divergente e confiabilidade da EABAP em estudantes do ensino fundamental II e ensino médio (Gomes, Golino, Pinheiro, Miranda, \& Soares, 2011)
Uma medida da abordagem profunda (AP) e da abordagem superficial (AS) foi obtida. A consistência interna dos itens é adequada, com alfa de 0,84 para $\mathrm{AP}$ e alfa de 0,75 para AS. A análise fatorial confirmatória mostra que o modelo de dois fatores que se correlacionam (AP e AS) é adequado para explicar a variância dos itens $\left(\chi^{2}=214,64\right.$; $g l=64 ; \mathrm{CFI}=0,95 ; \mathrm{RMSEA}=0,06$ ). São oito os itens marcadores da AS e nove itens marcadores da AP. Nenhum item marcador apresentou carga fatorial inferior a 0,45 . As análises foram elaboradas através do software Mplus 5.2. O escore fatorial gerado de AP e AS foi utilizado para a análise de dados das variáveis da questão de estudo.

\section{Análise de Dados}

Os escores fatoriais de Gf, AP e AS foram utilizados ao invés do procedimento de soma dos escores. A preferência pelos escores fatoriais deve-se ao fato de que eles incluem somente o escore verdadeiro e eliminam o erro e a variância específica. Escores somados, ao contrário, incluem o escore verdadeiro, o erro e a variância específica. Os escores fatoriais de Gf, AP e AS e as notas anuais em matemática, português, história e geografia foram padronizadas com média 100 e desvio-padrão 10 em cada série escolar. Esse procedimento permite analisar em uma mesma matriz de dados os escores fatoriais e as notas de todos os estudantes, mesmo de diferentes séries.

O modelamento por equação estrutural (MEE) foi usado para a análise e comparação das três hipóteses do estudo. Cada hipótese foi representada por um modelo na MEE. O ajuste aos dados de cada um dos três modelos foi verificado através do índice de ajuste comparativo (CFI) e da raiz quadrada média do erro de aproximação (RMSEA). CFI $>0,90$ e RMSEA $<0,08$ são usados neste estudo como critérios para indicar um adequado ajuste do modelo aos dados (Byrne, 2001).

Apesar dos dados coletados serem transversais e o estudo não empregar um desenho experimental ou longitudinal, o modelamento por equação estrutural permite ao pesquisador elaborar e testar hipóteses sobre relações causais entre variáveis latentes, como é o caso das hipóteses 1 e 2 do estudo. A análise causal via MEE, apesar de controversa e não aceita por alguns pesquisadores, é reconhecida como possível e correta tecnicamente, através do modelo causal de relações do MEE (Blunch, 2008).

Apesar da inteligência ser melhor medida através da aplicação de uma diversidade de testes capazes de mensurar várias habilidades cognitivas de diferentes domínios, este trabalho utilizou itens marcadores exclusivamente de Gf para medir inteligência. $\mathrm{O}$ uso circunscrito provém da necessidade de aplicar um número parcimonioso de instrumentos, de modo a viabilizar o estudo, e a preferência por itens marcadores de Gf provém das evidências de que a inteligência fluida é o melhor indicador da inteligência (Carroll, 1993). 


\section{Resultados}

\section{Estatística Descritiva}

Dados descritivos dos escores dos testes componentes do CTIF são apresentados na Tabela 1 . O Teste de Raciocínio Lógico (RL) mostra um aumento na média dos escores da $6^{\mathrm{a}}$ à $9^{\mathrm{a}}$ série, com um declínio no $1^{\mathrm{o}}$ ano do ensino médio, e um novo aumento a partir do $2^{\circ}$ ano. Os escores mínimo $(0,00)$ e máximo $(11,00)$ foram obtidos, no geral, por uma parcela pequena da amostra total $(0,3 \% \mathrm{e}$ $5,9 \%$ respectivamente). A média dos escores do Teste de Indução (I) aumenta no intervalo da $6^{\mathrm{a}}$ série ao $2^{\circ}$ ano e tem uma leve queda no $3^{\circ}$ ano. Apenas $1,7 \%$ da amostra total obteve escore igual à zero, enquanto $2,6 \%$ obtiveram o escore máximo $(8,00)$. Houve um aumento no escore médio no Teste de Raciocínio Geral (RG) entre a $6^{\mathrm{a}}$ e a $7^{\mathrm{a}}$ série, um declínio entre a $7^{\mathrm{a}}$ e $9^{\mathrm{a}}$ série, um novo aumento no $1^{\circ}$ e $2^{\circ}$ ano, seguido por um declínio no $3^{\circ}$ ano do ensino médio. Da amostra total, $3,8 \%$ obtiveram escore igual à zero, enquanto apenas $0,2 \%$ obtiveram escore igual à 8,00 . Os escores somados dos três testes geram o escore total bruto de Gf. Há um aumento gradual neste escore entre a $6^{\mathrm{a}}$ e a $9^{\mathrm{a}}$ série, com um declínio no $1^{\mathrm{o}}$ ano, e outro aumento no $2^{\circ}$ e $3^{\circ}$ ano do ensino médio. Pode-se perceber que nenhum dos participantes errou ou acertou todos os itens do escore bruto de Gf. O escore máximo obtido pela amostra total foi de 26 pontos e o mínimo foi 2 pontos, representando $0,2 \%$ e $0,3 \%$ da amostra do estudo. É importante relembrar que este estudo utiliza para a análise de dados o escore fatorial de Gf, obtido a partir do escore total bruto de Gf. Nesse sentido, a estatística descritiva do escore total bruto em Gf é tão ou mais importante, no presente estudo, do que o escore bruto de cada teste componente do CTIF, em separado.

Tabela 1

Tabela Descritiva por Série com Mínimo (mín.), Máximo (máx.) - Seguido pelo Percentual da Amostra que obteve esse Escore Específico -, Média (m) e Desvio-padrão (DP) dos Escores para Cada Um dos Testes de Inteligência Fluida: Raciocínio Lógico (RL), Raciocínio Indutivo (I), Raciocínio Geral (RG) e Gf, que é o Escore Somado de Cada Um dos Três Testes

\begin{tabular}{|c|c|c|c|c|c|c|c|c|c|c|c|c|c|c|c|}
\hline \multirow[t]{2}{*}{ Série } & \multicolumn{4}{|c|}{ RL } & \multicolumn{3}{|c|}{ I } & \multicolumn{4}{|c|}{ RG } & \multicolumn{4}{|c|}{ Gf } \\
\hline & Mín. & Máx. & $M$ & $D P$ & Mín. Máx. & $M$ & $D P$ & Mín. & Máx. & $M$ & $D P$ & Mín. & Máx. & $M$ & $D P$ \\
\hline $5^{\mathrm{a}}$ & $\begin{array}{c}0,00 \\
(2,6 \%)\end{array}$ & $\begin{array}{c}10,00 \\
(3,9 \%)\end{array}$ & 6,47 & 2,25 & $\begin{array}{cc}0,00 & 7,00 \\
(5,3 \%) & (3,9 \%)\end{array}$ & 2,68 & 1,65 & $\begin{array}{c}0,00 \\
(15,8 \%)\end{array}$ & $\begin{array}{c}5,00 \\
(2,6 \%)\end{array}$ & 1,27 & 1,76 & $\begin{array}{c}2,00 \\
(1,3 \%)\end{array}$ & $\begin{array}{c}21,00 \\
(1,3 \%)\end{array}$ & 10,92 & 3,77 \\
\hline $7^{\mathrm{a}}$ & $\begin{array}{c}2,00 \\
(1,0 \%)\end{array}$ & $\begin{array}{c}11,00 \\
(1,0 \%)\end{array}$ & 7,73 & 1,61 & $\begin{array}{cc}0,00 & 8,00 \\
(4,8 \%) & (1,0 \%)\end{array}$ & 3,35 & 1,75 & $\begin{array}{c}0,00 \\
(5,7 \%)\end{array}$ & $\begin{array}{c}6,00 \\
(1,9 \%)\end{array}$ & 1,34 & 2,54 & $\begin{array}{c}4,00 \\
(1,0 \%)\end{array}$ & $\begin{array}{c}23,00 \\
(1,0 \%)\end{array}$ & 13,63 & 3,33 \\
\hline $8^{\mathrm{a}}$ & $\begin{array}{c}2,00 \\
(1,2 \%)\end{array}$ & $\begin{array}{l}11,00 \\
(2,4 \%)\end{array}$ & 8,12 & 1,52 & $\begin{array}{cc}1,00 & 7,00 \\
(14,6 \%) & (4,9 \%)\end{array}$ & 3,52 & 1,77 & $\begin{array}{c}0,00 \\
(2,4 \%)\end{array}$ & $\begin{array}{c}6,00 \\
(1,2 \%)\end{array}$ & 1,32 & 3,07 & $\begin{array}{c}7,00 \\
(1,2 \%)\end{array}$ & $\begin{array}{l}21,00 \\
(2,4 \%)\end{array}$ & 14,72 & 3,15 \\
\hline $9^{a}$ & $\begin{array}{c}5,00 \\
(1,0 \%)\end{array}$ & $\begin{array}{l}11,00 \\
(3,9 \%)\end{array}$ & 8,61 & 1,21 & $\begin{array}{cc}1,00 & 8,00 \\
(5,8 \%) & (1,0 \%)\end{array}$ & 4,49 & 1,58 & $\begin{array}{c}0,00 \\
(2,9 \%)\end{array}$ & $\begin{array}{c}6,00 \\
(1,9 \%)\end{array}$ & 1,27 & 3,33 & $\begin{array}{c}8,00 \\
(1,9 \%)\end{array}$ & $\begin{array}{c}23,00 \\
(1,0 \%)\end{array}$ & 16,43 & 2,85 \\
\hline $1^{\circ}$ & $\begin{array}{c}2,00 \\
(1,1 \%)\end{array}$ & $\begin{array}{c}11,00 \\
(5,3 \%)\end{array}$ & 8,16 & 1,71 & $\begin{array}{cc}0,00 & 8,00 \\
(2,1 \%) & (1,1 \%)\end{array}$ & 4,55 & 1,72 & $\begin{array}{c}0,00 \\
(2,1 \%)\end{array}$ & $\begin{array}{c}7,00 \\
(1,1 \%)\end{array}$ & 1,49 & 3,20 & $\begin{array}{c}7,00 \\
(2,1 \%)\end{array}$ & $\begin{array}{c}23,00 \\
(1,1 \%)\end{array}$ & 15,91 & 3,35 \\
\hline $2^{\circ}$ & $\begin{array}{c}5,00 \\
(1,0 \%)\end{array}$ & $\begin{array}{c}11,00 \\
(7,0 \%)\end{array}$ & 8,57 & 1,29 & $\begin{array}{cc}1,00 & 8,00 \\
(2,0 \%) & (9,0 \%)\end{array}$ & 5,36 & 1,74 & $\begin{array}{c}1,00 \\
(4,0 \%)\end{array}$ & $\begin{array}{c}8,00 \\
(1,0 \%)\end{array}$ & 1,56 & 4,01 & $\begin{array}{c}10,00 \\
(1,0 \%)\end{array}$ & $\begin{array}{c}26,00 \\
(1,0 \%)\end{array}$ & 17,94 & 3,28 \\
\hline $3^{\circ}$ & $\begin{array}{c}5,00 \\
(2,1 \%)\end{array}$ & $\begin{array}{c}11,00 \\
(20,6 \%)\end{array}$ & 9,30 & 1,45 & $\begin{array}{cc}1,00 & 8,00 \\
(1,0 \%) & (5,2 \%)\end{array}$ & 5,34 & 1,44 & $\begin{array}{c}1,00 \\
(4,1 \%)\end{array}$ & $\begin{array}{c}7,00 \\
(9,3 \%)\end{array}$ & 1,43 & 4,28 & $\begin{array}{c}10,00 \\
(1,0 \%)\end{array}$ & $\begin{array}{l}26,00 \\
(1,0 \%)\end{array}$ & 18,92 & 3,22 \\
\hline Total & $\begin{array}{c}0,00 \\
(0,3 \%)\end{array}$ & $\begin{array}{c}11,00 \\
(5,9 \%)\end{array}$ & 8,19 & 1,76 & $\begin{array}{cc}0,00 & 8,00 \\
(1,7 \%) & (2,6 \%)\end{array}$ & 4,24 & 1,90 & $\begin{array}{c}0,00 \\
(3,8 \%)\end{array}$ & $\begin{array}{c}8,00 \\
(0,2 \%)\end{array}$ & 1,58 & 3,22 & $\begin{array}{c}2,00 \\
(0,2 \%)\end{array}$ & $\begin{array}{c}26,00 \\
(0,3 \%)\end{array}$ & 15,65 & 4,05 \\
\hline
\end{tabular}

A apresentação dos dados descritivos da EABAP encontra-se na Tabela 2. A média do escore bruto da abordagem profunda diminui entre a $6^{\mathrm{a}}$ e $9^{\mathrm{a}}$ série do ensino fundamental, e aumenta ao longo do ensino médio. O escore bruto mínimo foi de 13 pontos, enquanto o escore máximo foi de 45 pontos, sendo obtidos por $0,2 \%$ e $0,9 \%$ do total da amostra, respectivamente. Já a média do esco- re bruto da abordagem superficial diminui ao longo das séries investigadas nesse estudo. $\mathrm{O}$ escore mínimo obtido nessa abordagem foi de 7 pontos, enquanto o máximo foi 40 . Da amostra total, apenas $0,2 \%$ obteve o escore mínimo e outros $0,2 \%$ obtiveram o escore máximo nessa abordagem. 
Gomes, C. M. A. \& Golino, H. F. (2012). Validade Incremental da Escala de Abordagens de Aprendizagem (EABAP).

Tabela 2

Tabela Descritiva por Série com Mínimo (mín.), Máximo (máx.) - Seguido pelo Percentual da Amostra que obteve esse Escore Especifico -, Média (m) e Desvio-Padrão (DP) do Escore de Cada Uma das Abordagens de Aprendizagem

\begin{tabular}{lcccccccc}
\hline Série & \multicolumn{3}{c}{ Abordagem profunda } & \multicolumn{3}{c}{ Abordagem superficial } \\
\cline { 2 - 8 } & Mín. & Máx. & $M$ & $D P$ & Mín. & Máx. & $M$ & $D P$ \\
\hline $6^{\text {a }}$ & $17,00(1,3 \%)$ & $45,00(2,6 \%)$ & 33,09 & 6,88 & $10,00(1,3 \%)$ & $37,00(2,6 \%)$ & 23,99 & 5,93 \\
$7^{\text {a }}$ & $15,00(1,0 \%)$ & $45,00(2,9 \%)$ & 31,48 & 6,81 & $7,00(1,0 \%)$ & $38,00(1,0 \%)$ & 23,55 & 5,90 \\
$8^{\text {a }}$ & $16,00(1,2 \%)$ & $42,00(1,2 \%)$ & 30,77 & 5,75 & $10,00(1,2 \%)$ & $35,00(1,2 \%)$ & 23,34 & 4,93 \\
$9^{\text {a }}$ & $16,00(1,0 \%)$ & $43,00(1,9 \%)$ & 29,50 & 5,29 & $11,00(1,0 \%)$ & $37,00(1,0 \%)$ & 23,18 & 5,04 \\
$1^{\circ}$ & $13,00(1,1 \%)$ & $44,00(1,1 \%)$ & 30,01 & 6,34 & $10,00(1,1 \%)$ & $40,00(1,1 \%)$ & 22,40 & 5,95 \\
$2^{\circ}$ & $19,00(2,0 \%)$ & $44,00(1,0 \%)$ & 30,85 & 5,74 & $11,00(2,0 \%)$ & $34,00(1,0 \%)$ & 22,21 & 5,42 \\
$3^{\text {o }}$ & $17,00(1,0 \%)$ & $45,00(1,0)$ & 31,69 & 6,13 & $9,00(1,0 \%)$ & $37,00(2,1 \%)$ & 20,77 & 6,16 \\
\hline Total geral & $13,00(0,2 \%)$ & $45,00(0,9 \%)$ & 30,99 & 6,20 & $7,00(0,2 \%)$ & $40,00(0,2 \%)$ & 22,74 & 5,70 \\
\hline
\end{tabular}

\section{Modelagem}

O modelo 1 sustenta a hipótese de que a abordagem à aprendizagem apresenta um efeito direto explicativo das diferenças individuais frente à inteligência. Por sua vez, esse efeito gera um efeito indireto na explicação das diferenças individuais do rendimento escolar discente. A relação entre a abordagem à aprendizagem e o desempenho escolar nos conteúdos é mediado pela inteligência e pelo fator geral de desempenho acadêmico.

O modelo 2 representa a hipótese oposta à presente no modelo 1. A inteligência possui um efeito direto sobre a explicação da variância da abordagem à aprendizagem dos estudantes e esta explica diretamente o fator geral de desempenho acadêmico. Nesse sentido, a inteligência tem um papel de base na geração das diferenças individuais da abordagem à aprendizagem discente, da mesma forma que apresenta um efeito indireto na explicação das diferenças individuais de desempenho acadêmico, mediado pela abordagem à aprendizagem e pelo fator geral de desempenho acadêmico.

O modelo 3 sustenta uma hipótese distinta das anteriores. Nem a inteligência ou a abordagem à aprendizagem é posta em uma posição hierárquica de base capaz de explicar a variância da outra variável. Nenhuma delas atua como mediador da outra junto ao fator geral de desempenho acadêmico. $\mathrm{O}$ modelo 3 define que tanto a inteligência quanto a abordagem à aprendizagem apresentam efeitos diretos junto à variância do fator geral de desempenho acadêmico.

Tanto o modelo $1\left(\chi^{2}=95,33 ; g l=12 ; \mathrm{CFI}=0,97\right.$; RMSEA $=0,10)$ quanto o modelo $2\left(\chi^{2}=130,55 ; g l=12 ; \mathrm{CFI}=0,96\right.$; RMSEA $=0,12$ ) não têm um adequado grau de ajuste aos dados. Ambos apresentam um RMSEA inadequado (superior a 0,08 ), indicando que as hipóteses relacionadas a esses modelos devem ser rejeitadas. O modelo 3 possui um grau de ajuste adequado $\left(\chi^{2}=45,04 ; g l=11 ; \mathrm{CFI}=\right.$ 0,99 ; RMSEA $=0,07$ ), indicando que a hipótese dos efeitos diretos não somente é a mais adequada, mas é a única que não pode ser rejeitada.

\section{Validade Incremental}

Tomando para análise o modelo 3, poder-se-á, então, verificar a força explicativa da inteligência e da abordagem à aprendizagem sobre o fator geral de desempenho acadêmico. Visando obter uma estimativa mais robusta foi realizado um bootstraping de 1000 casos com intervalo de confiança de $90 \%$.

O fator geral de desempenho acadêmico é explicado em $25,40 \%(20,40 \%-31,20 \%)$ de sua variância pela inteligência e pela abordagem à aprendizagem. A carga fatorial da inteligência sobre o fator geral de desempenho acadêmico (FGDA) é de $0,35(0,29-0,41)$, indicando uma explicação de $12,25 \%(8,41 \%$ - 16,81\%) da variância desta variável latente. A carga fatorial da abordagem à aprendizagem sobre FGDA é de $0,29(0,23-0,36)$, indicando uma explicação de $8,64 \%(5,24 \%$ - 12,82\%) da variância desta variável latente.

Analisando a razão do poder preditivo da inteligência versus abordagem à aprendizagem, pode-se, na melhor das situações a favor da inteligência, apontar que esta possui uma razão de cerca de $3: 1$ sobre a abordagem à aprendizagem $(16,81 \%$ - limite superior da variância explicada pela inteligência -, dividido por 5,24\% - limite inferior da variância explicada pela abordagem à aprendizagem). Fora a melhor das situações apontadas entre as possíveis, deve-se apontar para uma razão de 1:1 entre inteligência e abordagem à aprendizagem, quanto ao seu poder preditivo sobre FGDA.

Os escores fatoriais da abordagem profunda e da abordagem superficial são fortemente explicados pela variável latente abordagem à aprendizagem. A abordagem profunda é explicada em $51,70 \%$ de sua variância $(41,00 \%$ $62,20 \%)$ e a abordagem superficial em $71,60 \%(59,40 \%$ $87,50 \%$ ) de sua variância. As diferenças individuais no desempenho dos conteúdos escolares são fortemente explicadas pelo fator geral de desempenho acadêmico. Matemática tem $65,40 \%(61,20 \%-69,10 \%)$ de sua variância explicada por esta variável latente. Português é explicado em $71,60 \%(68,10 \%-75,10 \%)$, Geografia em $83,10 \%$ 
$(80,20 \%-85,80 \%)$ e História em 85,40\% (82,70\% - 88, $10 \%)$ de sua variância pelo fator geral de desempenho acadêmico.

Inteligência e abordagem à aprendizagem apresentam uma correlação fraca de $0,22(0,16-0,29)$, e Matemática e Português também apresentam uma fraca correlação de $0,22(0,14-0,29)$.

\section{Discussão e Conclusão}

O presente trabalho buscou verificar o papel da abordagem à aprendizagem $(\mathrm{AbA})$ no desempenho escolar, além da inteligência. Três hipóteses foram elaboradas e empiricamente testadas por meio da modelagem por equações estruturais. A primeira hipótese sustenta que a AbA explica parte das diferenças individuais em inteligência, que por sua vez medeia a explicação do fator geral de desempenho acadêmico (FGDA), relegando à AbA um papel indireto. Já a segunda hipótese apresenta uma idéia oposta à da primeira hipótese, ou seja, a inteligência explica parte das diferenças individuais em $\mathrm{AbA}$, e possui um papel indireto na explicação de FGDA. A terceira hipótese, por sua vez, sustenta que não há mediação entre inteligência e $\mathrm{AbA}$ na explicação do FGDA. Inteligência e AbA explicariam FGDA, simultaneamente. Os resultados apontam que apenas a terceira hipótese não pode ser refutada, uma vez que é a única cujo modelo apresenta adequado ajuste aos dados empíricos $\left(\chi^{2}=45,04 ; g l=11 ; \mathrm{CFI}=0,99\right.$; RMSEA =0,07).

Vários trabalhos internacionais analisam o poder preditivo da $\mathrm{AbA}$, conjuntamente a outras variáveis psicológicas, tais como auto-eficácia (Fenollar et al., 2007; Liem et al., 2008; Senko \& Miles, 2008), personalidade (Furnham et al., 2009), estilos cognitivos e motivação (Diseth \& Martinsen, 2003). No entanto, esses estudos não verificam se a AbA possui algum poder explicativo adicional sobre o desempenho acadêmico, além das outras variáveis explicativas analisadas. A verificação da validade incremental de qualquer variável é um passo necessário aos estudos de validade preditiva. Na medida em que há indícios de que a AbA possui capacidade preditiva sobre a proficiência escolar, é importante verificar se esse construto ainda mantém o seu valor explicativo quando controlado o efeito de outras variáveis concorrentes, tal como a inteligência.

A inteligência é considerada como um dos construtos psicológicos de maior poder preditivo, se não o maior, em relação ao desempenho acadêmico (Deary et al., 2007; Gustafsson \& Undheim, 1996; Naglieri \& Bornstein, 2003; B. Spinath et al., 2006). Portanto, faz sentido verificar se AbA possui algum poder explicativo além da inteligência. Evidências favoráveis reforçariam e complementariam os estudos de validade preditiva da AbA, e salientariam a força de sua explicação sobre o desempenho acadêmico. Apesar de ser uma questão relevante para a área, poucos estudos buscaram claramente verificar a validade incremental da $\mathrm{AbA}$ em relação à inteligência, por meio de um tratamento de dados específico para esse problema. As evidências do presente trabalho corroboram os resulta- dos de Chamorro-Premuzic e Furnham (2008) e de Furnham et al. (2009), indicando que a AbA possui validade incremental na explicação do desempenho acadêmico, além da inteligência.

Este trabalho também evidenciou que a inteligência e a $\mathrm{AbA}$ se correlacionam fracamente $(0,22)$, reforçando resultados anteriores, como o de Chamorro-Premuzic e Furnham (2008), que encontram uma correlação pequena e significativa entre abordagem profunda e $\operatorname{Gf}(0,27)$, e o de Furnham et al. (2009), que mostraram correlações não significativas variando entre $-0,11$ e 0,13 entre testes de inteligência e as abordagens de aprendizagem. Interpretando esses resultados, do ponto de vista da medida, pode-se pensar, em um primeiro momento, que a baixa correlação entre inteligência e abordagens é um resultado positivo. Isso seria uma evidência favorável à validade de construto da $\mathrm{AbA}$, uma vez que ela mediria uma outra faceta da arquitetura cognitiva humana, que não a inteligência. No entanto, deve-se reconhecer que duas variáveis psicológicas não precisam apresentar uma correlação baixa para que suas identidades sejam configuradas como corretas. Em outas palavras, a baixa correlação entre variáveis é uma situação interessante, mas não necessária para a medida das mesmas.

Se do ponto de vista da medida a baixa correlação encontrada é um bom indicador, do ponto de vista educacional ela também pode ser concebida como positiva? A baixa correlação indica claramente que a adoção de interações profundas com os objetos de conhecimento escolar, relatada pelos alunos, tem pouca relação com a capacidade de pensar de forma abstrata, de estabelecer relações lógicas, e de resolver problemas novos, o que do ponto de vista educacional parece um contra-senso. Interagir mais profundamente com os objetos de conhecimento (abordagem profunda), segundo a teoria das abordagens, deveria propiciar uma ampliação na capacidade de pensar e aprender do estudante, ou seja, provocar alterações nas suas estruturas intelectuais (Biggs, 1985; Entwistle, 1997). O reverso também é verdadeiro, ou seja, uma maior capacidade de identificar relações lógicas, resolver problemas novos, etc, deveria propiciar ao estudante interações mais profundas com os objetos de conhecimento. Especulando, parece que a baixa correlação encontrada entre AbA e inteligência é uma situação negativa, do ponto de vista educacional, pois há uma considerável desconexão entre a maneira com que os estudantes relatam interagir com os objetos de conhecimento de natureza escolar e sua capacidade de resolver problemas, estabelecer relações, e resolver problemas novos (Gf). O ideal seria que esses dois aspectos fossem integrados, de modo a potencializar o desenvolvimento dos alunos.

Algumas conclusões podem ser elaboradas a partir dos resultados do estudo. Elas serão sintetizadas a seguir, e organizadas em três pontos principais:

1. A abordagem à aprendizagem possui validade incremental. O papel preditivo da abordagem à aprendizagem sobre o fator geral de desempenho acadêmico é 
igual à inteligência ou, na pior das condições, cerca de três vezes menor do que esta.

2. Nem a abordagem à aprendizagem é causa da inteligência, nem a inteligência é causa da abordagem à aprendizagem, no que diz respeito ao seu papel preditivo frente às diferenças individuais do desempenho escolar. Ambas as variáveis têm efeito direto na explicação do fator geral de desempenho acadêmico, que explica diretamente as diferenças individuais nos conteúdos escolares.

3. A correlação fraca entre inteligência e abordagem à aprendizagem indica que ambas as variáveis são relativamente independentes, mas não separadas uma da outra. Apresentar maior inteligência não indica uma forte propensão a apresentar uma abordagem à aprendizagem mais profunda. Conforme indica a literatura, a abordagem à aprendizagem é uma articulação imbricada entre estratégias e motivações conscientes sobre como interagir com os objetos do conhecimento. Pessoas que apresentam maior inteligência podem ou não possuir estratégias e/ou motivações conscientes profundas no que tange aos objetos de conhecimento escolares. Estudos futuros necessitam investigar o papel do contexto e da história de desenvolvimento individual junto aos tipos de relação possíveis entre a inteligência e a abordagem à aprendizagem. É possível que diferentes relações se formem em diferentes trajetórias de desenvolvimento, influenciadas por contextos distintos.

Algumas limitações das evidências obtidas provém do próprio limite da amostra. Os participantes do estudo são de uma única escola, com um perfil delimitado. As evidências necessitam, pois, ser encontradas em amostras distintas, com diferentes níveis socioeconômicos e outras variáveis sociodemográficas. Por outro lado, a amostra empregada apresenta uma diversidade considerável, no que tange à idade e ao nível de escolaridade, possibilitando uma boa variabilidade de casos. Nesse sentido, a amostra do estudo é homogênea em algumas características e heterogênea em outras, apresentando pontos fortes e fracos.

Implicações podem ser obtidas a partir das evidências encontradas:

1. A abordagem à aprendizagem não se reduz à inteligência. Compreender a dinâmica de desenvolvimento entre $\mathrm{a}$ abordagem à aprendizagem e $\mathrm{a}$ inteligência é uma agenda importante para o futuro das pesquisas na área.

2. Se, possivelmente, a abordagem à aprendizagem é tão importante quanto à inteligência para explicar as diferenças individuais do desempenho escolar, então é relevante considerar a potencialidade de intervenções psicoedutivas junto ao modo como os estudantes interagem com os objetos de conhecimento escolares.

3. Uma questão educacional relevante envolve compreender se é mais adequado intervir junto à inteligência ou intervir junto à abordagem à aprendiza- gem, para alterar e ampliar o desenvolvimento da capacidade de aprender dos estudantes. Por outro lado, intervenções mistas podem ser mais eficientes, de modo que compreender como a alteração de uma variável afeta e interfere no desenvolvimento da outra variável é um desafio para futuras pesquisas.

4. Foi surpreendente verificar que o campo de estudos em abordagem à aprendizagem não seja pródigo em estudos que investigam a validade incremental desta variável psicológica. Para o desenvolvimento da psicologia, enquanto ciência e profissão, é necessário que as diferentes tradições em psicologia tentem se conectar e se envolver em questões desafiadoras que abram novas possibilidades de ação e investigação.

Em suma, o presente trabalho apresenta evidências favoráveis à validade incremental da abordagem de aprendizagem, em relação à inteligência, na explicação do desempenho acadêmico. Os resultados apontam que ambas as variáveis estão em um mesmo nível hierárquico, ou seja, uma não medeia a outra no caminho de explicação do rendimento acadêmico e apresentam baixa correlação entre si.

\section{Referências}

Alexander, J. M., Johnson, K. E., Albano, J., Freygang, T., \& Scott, B. (2006). Relations between intelligence and the development of metaconceptual knowledge. Metacognition and Learning, 1, 51-67.

Alexander, J. M., Johnson, K. E., Leibham, M. E., \& DeBauge, C. (2004). Constructing domain-specific knowledge in kindergarten: Relations among knowledge, intelligence, and strategic performance. Learning and Individual Differences, $15,35-52$

Almeida, L. S. (1992). Inteligência e aprendizagem: Dos seus relacionamentos à sua promoção. Psicologia: Teoria e Pesquisa, 8(3), 277-292.

Almeida, L. S. (1996). Cognição e aprendizagem: Como a sua aproximação conceptual pode favorecer o desempenho cognitivo e a realização escolar. Psicologia: Teoria, Investigação e Prática, 1, 17-32.

Baddeley, A. (1968). A 3 min reasoning test based on grammatical transformation. Psychonomic Science, 10, 341-342.

Beier, M. E., Campbell, M., \& Crook, A. E. (2010). Developing and demonstrating knowledge: Ability and non-ability determinants of learning and performance. Intelligence, 38(1), 179-186.

Biggs, J. B. (1978). Individual and group differences in study processes. British Journal of Educational Psychology, 48, 266279.

Biggs, J. B. (1985). The role of meta-learning in study processes. British Journal of Educational Psychology, 55, 185-212.

Biggs, J. B. (1987a). The Learning Process Questionnaire (LPQ): Users' manual. Hawthorn, Australia: Australian Council for Educational Research.

Biggs, J. B. (1987b). The Study Process Questionnaire (SPQ). Manual. Hawthorn, Australia: Australian Council for Educational Research.

Biggs, J., Kember, D., \& Leung, D. P. (2001). The revised twofactor Study Process Questionnaire: R-SPQ-2F. British Journal of Educational Psychology, 71(1), 133-149. 
Biggs, J., \& Tang, C. (2007). Teaching for quality learning at university. New York: McGraw-Hill.

Blunch, N. J. (2008). Introduction to structural equation modelling using SPSS and AMOS. Thousand Oaks, CA: SAGE.

Boruchovitch, E. (1993). A Psicologia Cognitiva e a metacognição: Novas perspectivas para o fracasso escolar brasileiro. Tecnologia Educacional, 22(110/111), 22-28.

Boruchovitch, E. (1999). Estratégias de aprendizagem e desempenho escolar: Considerações para a prática educacional. Psicologia: Reflexão e Crítica, 12(2), 361-373.

Brody, N. (1997). Intelligence, schooling, and society. American Psychologist, 52(10), 1046-1050.

Byrne, B. (2001). Structural equation modeling with AMOS: Basic concepts, applications and programming. Mahwah, NJ: Lawrence Erlbaum.

Carroll, J. B. (1993). Human cognitive abilities: A survey of factor analytic studies. New York: Cambridge University Press.

Case, J. M., \& Marshall, D. (2009). Approaches to learning. In M. Tight, K. H. Mok, J. Huisman, \& C. C. Morphew (Eds.), The Routledge internacional handbook of higher education (pp. 9-22). New York: Routledge.

Ceci, S. J. (1991). How much does schooling influence general intelligence and its cognitive components? A reassessment of the evidence. Developmental Psychology, 27, 703-722.

Chamorro-Premuzic, T., \& Furnham, A. (2008). Personality, intelligence and approaches to learning as predictors of academic performance. Personality and Individual Differences, 44(7), 1596-1603.

Colom, R., \& Flores-Mendoza, C. E. (2007). Intelligence predicts scholastic achievement irrespective of SES factors: Evidence from Brazil. Intelligence, 35(3), 243-251.

Costa, P. T., Jr., \& McCrae, R. (1992). Revised NEO Personality Inventory (NEO-PI-R) and NEO Five-factor Inventory (NEOFFI): Professional manual. Odessa, FL: Psychological Assessment Resources.

Da Silva, A. L., \& Sá, I. (1993). Saber estudar e estudar para saber: Ciências da Educação. Porto, Portugal: Porto.

Deary, I. J., \& Johnson, W. (2010). Intelligence and education: Causal perceptions drive analytic processes and therefore conclusions. International Journal of Epidemiology. Advance online publication. doi: 10.1093/ije/dyq072

Deary, I. J., Strand, S., Smith, P., \& Fernandes, C. (2007). Intelligence and educational achievement. Intelligence, 35(1), 13-21.

Diseth, A. (2001). Validation of a Norwegian version of the Approaches and Study Skills Inventory for Students (ASSIST): Application of structural equation modelling. Scandinavian Journal of Educational Research, 45, 381-394.

Diseth, A. (2002). The relationship between intelligence, approaches to learning and academic achievement. Scandinavian Journal of Educational Research, 46(2), 219-230.

Diseth, Å., \& Martinsen, Ø. (2003). Approaches to learning, cognitive style, and motives as predictors of academic achievement. Educational Psychology, 23(2), 195-207.

Entwistle, N. J. (1988). Motivational factors in students' approaches to learning. In R. R. Schmeck (Ed.), Learning strategies and learning styles (pp. 21-51). New York: Plenum Press.

Entwistle, N. J. (1997). The Approaches and Study Skills Inventory for Students (ASSIST). Edinburgh, UK: Centre for Research on Learning and Instruction, University of Edinburgh.
Entwistle, N., McCune, V., \& Walker, P. (2001). Conceptions, styles, and approaches within higher education: Analytical abstractions and everyday experience. In R. J. Sternberg \& L. Zhang (Eds.), Perspectives on thinking, learning, and cognitive styles (pp. 103-136). Mahwah, NJ: Lawrence Erlbaum.

Entwistle, N. J., Meyer, J. H. F., \& Tait, H. (1991). Students failure: Disintegrated perceptions of studying and the learning environment. Higher Education 21, 249-261.

Entwistle, N. J., \& Ramsden, P. (1983). Understanding student learning. London: Croom Helm.

Fenollar, P., Román, S., \& Cuestas, P. J. (2007). University students' academic performance: An integrative conceptual framework and empirical analysis. British Journal of Educational Psychology, 77, 873-891.

Freudenthaler, H. H., Spinath, B., \& Neubauer, A. C. (2008). Predicting school achievement in boys and girls. European Journal of Personality, 22 (3), 231-245.

Fry, H., Ketteridge, S., \& Marshall, S. (2009). Understanding student learning. In H. Fry, S. Ketteridge, \& S. Marshall (Eds.), $A$ handbook for teaching and learning in higher education (pp. 8-22). New York: Routledge.

Fuentes, V. L. P., Lima, R., \& Guerra, D. S. (2009). Atitudes em relação à Matemática em estudantes de Administração. Psicologia Escolar e Educacional, 13(1), 133-141.

Furnham, A., Monsen, J., \& Ahmetoglu, G. (2009). Typical intellectual engagement: Big Five personality traits, approaches to learning and cognitive ability predictors of academic performance. British Journal of Educational Psychology, 79(4), 769-782.

Gagné, F., \& St. Père, F. (2001). When IQ is controlled, does motivation still predict achievement? Intelligence, 30, 71-100.

Garner, R., Hare, V. C., Alexander, P., Haynes, J., \& Winograd, P. (1984). Inducing use of a text lookback strategy among unsucessful readers. American Educational Research Journal, 21, 789-798.

Goff, M., \& Ackerman, P. L. (1992). Personality-intelligence relations: Assessment of typical intellectual engagement. Journal of Educational Psychology, 84, 537-553.

Gomes, C. F. (2005). Escala de avaliação de processos de estudo (EAPE). In Psicologia.com.pt - O Portal dos Psicólogos. Retrieved September 22, 2008, from http:// www.psicologia.com.pt

Gomes, C. M. A., \& Borges, O. (2009). Qualidades psicométricas do conjunto de Testes de Inteligência Fluida. Avaliação Psicológica, 8(1), 17-32.

Gomes, C. M. A., Golino, H. F., Pinheiro, C. A. R., Miranda, G. R., \& Soares, J. (2011). Validação da Escala de Abordagens de Aprendizagem (EABAP) em uma amostra brasileira. Psicologia: Reflexão e Crítica, 24(1), 1-9.

Gustafsson, J. E., \& Undheim, J. O. (1996). Individual differences in cognitive functions. In D. C. Berliner \& R. C. Calfee (Eds.), Handbook of educational psychology (pp. 186-242). New York: Prentice Hall International.

Hattie, J., Biggs, J., \& Purdue, N. (1996). Effects of learning skills interventions on students: A meta analysis. Review of Educational Research, 66(2), 99-136.

Irving, P, Cammock, T., \& Lynn, R. (2001). Some evidence for the existence of a general factor of semantic memory and its components. Personality and Individual Differences, 30, 857-871

Lajoie, S. P. (2008). Metacognition, self-regulation, and selfregulated learning: A rose by any other name? Educational Psychological Review, 20, 469-475. 
Liem, A. D., Lau, S., \& Nie, Y. (2008). The role of self-efficacy, task value, and achievement goals in predicting learning strategies, task disengagement, peer relationship, and achievement outcome. Contemporary Educational Psychology, 33, 486512.

Light, G., Cox, R., \& Calkins, S. (2009). Learning and teaching in higher education: The reflective professional. London: Sage.

Marton, F., \& Säljö, R. (1976a). On qualitative differences in learning: I - Outcome and process. British Journal of Educational Psychology, 46, 4-11.

Marton, F., \& Säljö, R. (1976b). On qualitative differences in learning: II. Outcome as a function of the learner's conception of the task. British Journal of Educational Psychology, 46, $115-127$

Mønnesland, K. (1985). Intelligensprøver for Voksne. Oslo, Norway: TANO.

Naglieri, J. A., \& Bornstein, B. T. (2003). Intelligence and achievement: Just how correlated are they? Journal of Psychoeducational Assessment, 21, 244-260.

Neisser, U., Boodoo, G., Bouchard, T. J. J., Boykin, A. W., Brody, N., \& Ceci, S. J. (1996). Intelligence: Knowns and unknowns. American Psychologist, 51, 77-101.

Oliver, B. (2007). What is quality university learning and how might microlearning help to achieve it? In T. Hug (Ed.), Didactics of microlearning: Concepts, discourses and examples (pp. 365-380). Münster, Germany: Waxmann.

Paasche, A. (1957). Rettleiing for Bruk av A-Batteriets Prøver for Mekanisk-Teknisk Innsikt. Oslo, Norway: Arbeidsdirektoratet.

Pasquali, L. (2007). Validade dos testes psicológicos: Será possível reencontrar o caminho? Psicologia: Teoria e Pesquisa, 23, 99-107.

Pressley, M., \& Levin, J. R. (1983). Cognitive strategy research: Psychological Foundations. New York: Springer-Verlag.

Riding, R., \& Rayner, S. (Eds.). (1998). Cognitive styles and learning strategies: Understanding style differences in learning and behaviour. London: David Fulton.

Robinson, A., \& Udall, M. (2006). Using formative assessment to improve student learning through critical reflection. In C. Bryan \& K. Clegg (Eds.), Innovative assessment in higher education (pp. 92-99). New York: Routledge.

Rosário, P., Almeida, L., Núñez, J. C., \& González-Pienda, J. A. (2004). Abordagem dos alunos à aprendizagem: Análise do construto. Psico-USF, 9(2), 117-127.

Schunk, D. H. (2008). Metacognition, self-regulation, and selfregulated learning: Research recommendations. Educational Psychological Review, 20, 463-467.

Schwinger, M., Steinmayr, R., \& Spinath, B. (2009). How do motivational regulation strategies affect achievement: Mediated by effort management and moderated by intelligence. Learning and Individual Differences, 19, 621-627.

Senko, C., \& Miles, K. M. (2008). Pursuing their own learning agenda: How mastery-oriented students jeopardize their class performance. Contemporary Educational Psychology, 33, $561-583$

Souza, N. A., \& Boruchovitch, E. (2009). Avaliação da aprendizagem e motivação para aprender: Tramas e entrelaços na formação de professores. Educação Temática Digital, 10, 204-227.

Spinath, B., Spinath, F. M., Harlaar, N., \& Plomin, R. (2006). Predicting school achievement from general cognitive ability, self-perceived ability, and intrinsic value. Intelligence, 34, 363-374.
Spinath, F. M., Spinath, B., \& Plomin, R. (2008). The nature and nurture of intelligence and motivation in the origins of sex differences in elementary school achievement. European Journal of Personality, 22, 211-229.

Steinmayr, R., \& Spinath, B. (2009). The importance of motivation as a predictor of school achievement. Learning and Individual Differences, 19, 80-90.

Stel, M. van der, \& Veenman, M. V. J. (2008). Relation between intellectual ability and metacognitive skillfulness as predictors of learning performance of young students performing tasks in different domains. Learning and Individual Differences, $18,128-134$.

Struyven, K., Dochy, F., Janssens, S., \& Gielen, S. (2006). On the dynamics of students' approaches to learning: The effects of the teaching/learning environment. Learning and Instruction, 20, 1-16.

Veenman, M. V. J., \& Beishuizen, J. J. (2004). Intellectual and metacognitive skills of novices while studying texts under conditions of text difficulty and time constraint. Learning and Instruction, 14, 621-640.

Veenman, M. V. J., Kerseboom, L., \& Imthorn, C. (2000). Test anxiety and metacognitive skillfulness: Availability versus production deficiencies. Anxiety, Stress and Coping, 13, 391412.

Veenman, M. V. J., \& Spaans, M. A. (2005). Relation between intellectual and metacognitive skills: Age and task differences. Learning and Individual Differences, 15, 159-176.

Vermunt, J., \& Vermetten, Y. (2004). Patterns in student learning: Relationships between learning strategies, conceptions of learning and learning orientations. Educational Psychology Review, 16, 359-384.

Wadhwa, S. (2008). A handbook of teaching and learning. New Delhi, India: SARUP \& SONS.

Watkins, D. A. (2007). Comparing ways of learning. In M. Bray, B. Adamson, \& M. Mason (Eds.), Comparative education research: Approaches and methods (pp. 299-314). Hong Kong, People's Republic of China: Springer.

Watkins, M. W., Lei, P. W., \& Canivez, G. L. (2007). Psychometric intelligence and achievement: A cross-lagged panel analysis. Intelligence, 35, 59-68.

Weinstein, C. E., \& Mayer, R. E. (1985). The teaching of learning strategies. In M. Wittrock (Ed.), Handbook of research on teaching ( $3^{\text {rd }}$ ed., pp. 1-53). New York: Macmillan.

Wechsler, D. (1997). Wechsler Adult Intelligence Scale: Administration and scoring manual ( $3^{\text {rd }}$ ed.). San Antonio, TX: The Psychological Corporation.

Wild, M., \& Quinn, C. N. (1998). Implications of educational theory for the design of instructional multimedia. British Journal of Educational Technology, 29, 73-82.

Wonderlic, E. (1992). Wonderlic personnel test. Libertyville, IL: Wonderlic.

Yu, C., Williams, A., Lin, C. F., \& Yu, W.-C. (2010). Revisit planning effective multimedia instructions. In H. Song \& T. Kidd (Eds.), Human performance and instructional techonology (pp. 131-148). Hershey, PA: IGI Global. 